\title{
Influencia térmica e higroscópica en la permitividad relativa de material cerámico crudo utilizado en el proceso de fabricación de baldosas para piso
}

\author{
O. RODRÍGUEZ P. ${ }^{1}$, W. ROMERO ${ }^{2}$, J. A. VEGA ${ }^{3}$, S. ESGUERRA ${ }^{4}$ \\ Universidad Central. Escuela de Ingeniería. Facultad de Ingeniería Electrónica. Grupo de Estado Sólido y Optoelectrónica. Bogotá, Colombia \\ ${ }^{1}$ Fisico. Ms.C. En Física Nuclear. Director Grupo de Estado Sólido y Optoelectrónica \\ ${ }^{2}$ Ingeniero Electrónico. Profesor Auxiliar de Investigación. \\ ${ }^{3}$ Ingniero Electricista. Ms.C en Ingeniería Eléctrica. Profesor Auxiliar de Investigación. \\ ${ }^{4}$ Estudiante $7^{\circ}$ Semestre Ingeniería Electrónica. Auxiliar de Investigación.
}

\begin{abstract}
En el presente trabajo se demuestra el comportamiento térmico e higroscópico de muestras de material cerámico crudo utilizadas en el proceso de fabricación de baldosas para piso. El trabajo experimental demostró que la presión de prensado no es un factor tan relevante como la humedad relativa $\mathrm{H}$, en el comportamiento de las muestras a la acción directa de la luz y su influencia en la permitividad eléctrica relativa.
\end{abstract}

Palabras Claves: Permitividad eléctrica relativa, Higroscopía, Función paramétrica, Humedad relativa.

Thermal influence and capacity of absorsion of water in the relative permitivity of raw ceramic material used in the process of production of tiles for floor.

In the presently work is demonstrated the thermal behavior and the degree of absorption of water for sample of raw ceramic material used in the process of production of tiles for floor. The experimental work demonstrated that the pressure of having pressed is not such an excellent factor as the relative humidity $\mathrm{H}$, in the behavior of the samples to the direct action of the light and their influence in the relative electric permitivity.

Key words: Relative electric Permitivity, parametric Function, relative Humidity.

\section{INTRODUCCIÓN}

En el proceso de fabricación de baldosas para piso cerámico y en muchos otros casos, relacionados con el estudio y caracterización de material arcilloso, surge la necesidad de hacer controles previos de los parámetros más relevantes como: a) grado de humedad ${ }^{1}$ de las muestras; b) temperatura de cocido; c) presión de prensado y d) densidad aparente (DAP), por enunciar algunas. Dichos controles se hacen sobre muestras crudas por diferentes métodos, como el más reciente ${ }^{2}$, con el objetivo de determinar el comportamiento de propiedades eléctricas como: a) conductividad; b) permitividad eléctrica; c) dimensiones de grano ${ }^{3}$, y otras.

En el presente trabajo se muestran los resultados experimentales del comportamiento de absorción y emisión térmica de más de 100 muestras de material cerámico crudo (probetas) cuya composición química y grado de humedad relativa se detalla en la tabla 1.

La propiedad eléctrica más importante que tienen los materiales dieléctricos es la permitividad eléctrica $\varepsilon$, que para compuestos arcillosos es compleja, puesto que su cálculo y determinación es un problema de múltiples fases (aire, agua, sólido), tal y como lo reporta ${ }^{4}$. Un modelo físico riguroso que describa el comportamiento de la permitividad eléctrica relativa $\varepsilon$, para esta clase de sistemas atómicos, es extenso y requiere de una buena máquina de cálculo y bastante tiempo disponible, sin
TABLA 1. COMPOSICIÓN QUÍMICA DEL MATERIAL CERÁMICO UTILIZADO.

\begin{tabular}{|c|c|}
\hline Componentes & Composición por masa \% \\
\hline $\mathrm{Si}_{2} \mathrm{O}_{3}$ & 65 \\
\hline $\mathrm{Na}_{2} \mathrm{O}$ & 10 \\
\hline $\mathrm{Fe}_{2} \mathrm{O}_{3}$ & $4{ }_{-} 5$ \\
\hline $\mathrm{K}_{2} \mathrm{O}$ & $2{ }_{-} 3$ \\
\hline $\mathrm{Al}_{2} \mathrm{O}_{3}$ & 2 \\
\hline $\mathrm{B}$ & $<1$ \\
\hline Otros & 14 \\
\hline
\end{tabular}

La humedad relativa de base fue de 5 _ $7 \%$

embargo, el método experimental es una buena herramienta que tiene los científicos para determinar el valor más aproximado al valor teórico de este parámetro fundamental.

Existen modelos empíricos de determinación no solo, de $\varepsilon$, sino de la conductividad eléctrica $\sigma$, susceptibilidad eléctrica $\chi$ y densidad aparente DAP, para compuestos multifase, como para el caso que nos ocupa.

Acorde con los datos experimentales, los principios básicos de la teoría electromagnética, la forma de absorción y emisión de la luz por los cuerpos, se estableció una relación entre el grado de humedad de las probetas objeto de estudio, la temperatura y la permitividad eléctrica relativa $\varepsilon$. 


\section{PROCEDIMIENTO EXPERIMENTAL}

\subsection{Etapa de preparación.}

Se tomaron 10 grupos, cada uno de 20 probetas de material cerámico crudo, con una participación de 5 gr de $\mathrm{H}_{2} \mathrm{O}$ por cada 100 gr de muestra y con una humedad relativa $\mathrm{H}$ entre el 5 y $7 \%$ de base húmeda. El rango de presión de prensado utilizado en el proceso de fabricación de las muestras, fue de 12 a 15 Bar.

Cada probeta fue cuidadosamente perforada con 4 electrodos diametralmente opuestos (como se muestra en la figura 1), que sirvieron de base para registrar voltaje inducido $\mathrm{V}_{\text {in }} \mathrm{y}$ corriente inducida $\mathrm{I}_{\text {in }}$ simultáneamente. A cada una de las muestras se le aplicó una capa de barniz negro mate, para lograr un mayor grado de absorción de luz. Después fueron encerradas en una cámara de paredes internas reflectivas forradas en aluminio, con sensores adaptados adecuadamente para tal caso, como se muestra en la figura 2.

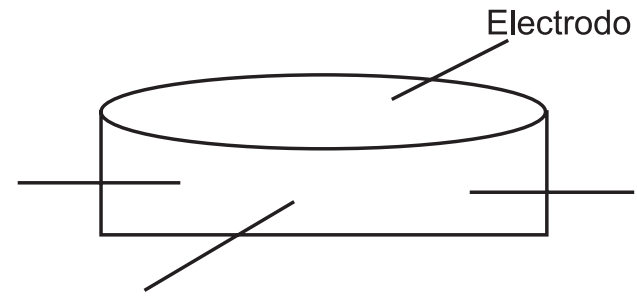

Figura 1. Disposición de los electrodos en las muestras de material cerámico crudo.

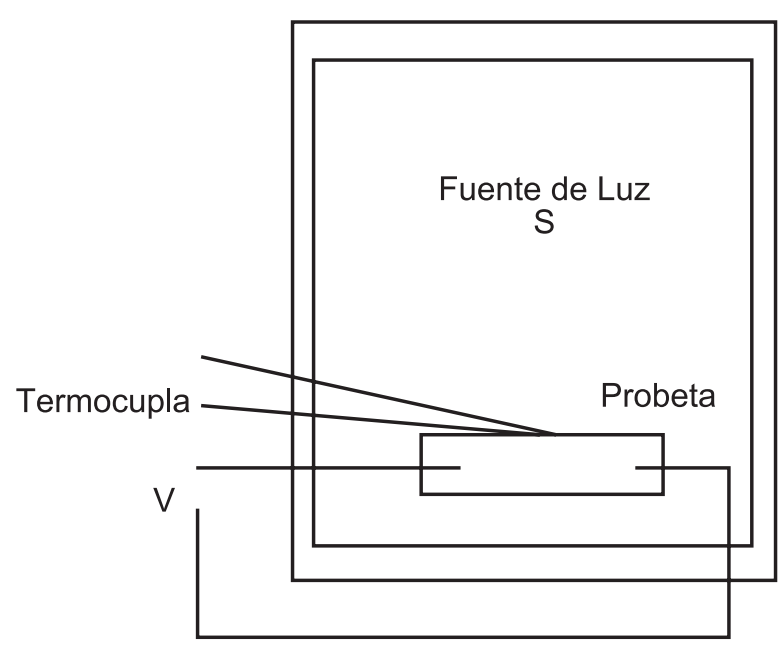

Figura2. Cámara de pruebas de absorción y emisión de luz.

La fuente de luz utilizada fue, luz alógena de 150 Watts de potencia, una termocupla tipo k, un nano amperímetro y un sistema electrónico de registro y control de datos, para generar la base correspondiente de los mismos y graficar las curvas necesarias. Las dimensiones de las probetas utilizadas en los experimentos, estuvieron dentro de los siguientes rangos: espesor h (de 0.8 a $0.85 \mathrm{~cm}$ ); área superficial S (de 11.30 a 12.56 $\left.\mathrm{cm}^{2}\right)$.

\subsection{Medición de parámetros térmicos}

Al conectar la fuente de luz alógena de 150 Watts, se activaron automáticamente los sensores de temperatura, voltaje y corriente. Cada 30 segundos, se registraron los datos correspondientes, hasta llegar a una temperatura máxima de $370^{\circ}$ $\mathrm{K}$, luego se apagó la fuente de luz y se siguieron tomando los datos de descenso.

\section{DISCUSIÓN DE RESULTADOS}

\subsection{Dependencia de la permitividad eléctrica relativa $\varepsilon_{\mathrm{r}}$ de la humedad relativa $\mathrm{H}$.}

El comportamiento eléctrico de un material aislante ideal, está descrito por el grado de polarización $\mathrm{P} \rightarrow$ que presenta la muestra a la acción directa de un campo eléctrico $\mathrm{E} \rightarrow$ invariante en el tiempo, se puede definir como ${ }^{1}$ :

$$
\vec{P}=\vec{P}_{d}+\vec{P}_{m}
$$

donde: $\rightarrow \mathrm{P}_{\mathrm{d}}$ - polarización de las muestras en la primera etapa de secado; $\rightarrow \mathrm{P}_{\mathrm{m}}$ - polarización del material en dependencia de la humedad relativa $\mathrm{H}$.

Sí en una unidad de volumen se formaran $\mathrm{N}$ dipolos, y se generara a su vez un campo local interno $\rightarrow \mathrm{E}^{\prime}$, entonces:

$$
\vec{P}_{m}=H N_{m} \alpha_{m} \vec{E}^{\prime}
$$

donde: $\alpha_{\mathrm{m}}$ - polarizabilidad molecular; $\mathrm{N}_{\mathrm{m}}$ - número de dipolos por unidad de volumen.

Recurriendo a la relación:

$$
\frac{P_{d}}{P_{m}}=\frac{b(f)-1}{H a(f)}
$$

La polarización en la ecuación (1), puede ser expresada en términos de una sola variable, es decir de $\rightarrow \mathrm{P}_{\mathrm{m}^{\prime}}$, por lo que (1) será entonces:

$$
\vec{P}=\vec{P}_{m}\left(1+\frac{b(f)-1}{h a(f)}\right)
$$

donde: $a(f)$ y $b(f)$ - son funciones dependientes de la frecuencia de oscilación de los dipolos formados en el volumen del material cerámico crudo y que además dependen fuertemente del factor estructural de la red del material cerámico.

Recurriendo a los principios básicos del fenómeno de polarización de los dieléctricos, en presencia de un campo eléctrico externo, el vector de polarización $\mathrm{P} \rightarrow$ se puede calcular como $^{5}$ :

$$
\vec{P}=\vec{P}_{m}\left(1+\frac{b(f)-1}{h a(f)}\right)
$$

donde: $\mathrm{k}$ - constante dieléctrica del material en cuestión; $\varepsilon_{\mathrm{o}}$ - permitividad eléctrica del vacío; $\mathrm{E} \rightarrow$ - campo eléctrico externo.

Igualando las ecuaciones (4) y (5), se tiene entonces:

$$
\varepsilon_{r}=\varepsilon_{o}+\frac{P_{m}}{E}\left(H+\frac{b(f)-1}{a(f)}\right)
$$

El coeficiente de proporcionalidad (7), se puede definir como la constante higroscópica del material cerámico crudo, 
que determina el grado de humedad que puede contener la muestra después del proceso de sacado.

$$
\left(H+\frac{b(f)-1}{a(f)}\right)=\gamma
$$

Consecuencia de lo anterior, la permitividad eléctrica relativa está pues, fuertemente influenciada por el parámetro $\gamma$

\subsection{Dependencia térmica}

En esta segunda etapa del trabajo experimental, los resultados obtenidos del comportamiento de las probetas al aumento de la temperatura hasta los $370^{\circ} \mathrm{K}$ y su descenso posterior, se detallan en las siguientes figuras 3,4 y 5, que muestran respectivamente los dos tipos de procesos que se presentan con el material cerámico crudo, cuando el grado de humedad $\mathrm{H}$ varía.

Las dos curvas presentadas en el gráfico 1, caracterizan el comportamiento térmico de las probetas objeto de estudio con relación al voltaje inducido, registrado por sistema de control establecido para tal fin. Hay que resaltar el hecho de que el pico de absorción térmica presenta su máximo valor alrededor de los $343^{\circ} \mathrm{K}$ para todas las muestras utilizadas en el desarrollo del presente trabajo de investigación.

Los valores presentados a la derecha de las curvas en la figura 4, corresponden a los calculados para las funciones paramétricas $a(f)$ y $b(f)$.

En la figura 5 se comparan los resultados experimentales con el comportamiento teórico de la corriente inducida en las muestras de material cerámico con relación a la temperatura.

\section{CONCLUSIONES.}

El comportamiento mostrado en la gráfica 3, demuestra una vez mas que los procesos acaecidos en el interior de las probetas son de tipo probabilístico y que por esta razón se ajusta a la teoría establecida por Maxwell-Boltzman para radiación de cuerpo negro.

Es fuerte la influencia del parámetro $\gamma$, calculado en la ecuación (6), en la permitividad eléctrica relativa, pues las curvas obtenidas en la figura 4 demuestran este hecho y conducen directamente al cálculo de las funciones paramétricas $a(f) y b(f)$.

\section{BIBLIOGRAFÍA}

1. Ang $\mathrm{Hu}$ and Yonghan Fang, et al. Humidity Dependence of Apparent Dielectric Constant for DSP Cement Materials at High Frequencies. J. Am. Ceram. Soc. 82 [7] 1741 - 47 (1999)

2. Marchetti, B. y Marco Revel, G. Medida en línea de la densidad en crudo de baldosas cerámicas. Análisis de incertidumbre. Qualicer 2002. Casteelón España. Pp P.GI - $13-22$

3. Dale P. Bentz. et al. Influence of Cement Particle - Size Distribution on Early Age Autogenous Strains and Stresses in Cement - Based Materials. J. Am. Ceram. Soc. 84 [1] 129 - 35 (2001)

4. Maerz, H., Chepur, P., Myers, J., Linz, J. Concrete Roughness Characterization Using Laser Profilometry for Fiber - Reinforced Polymer Sheet Application. Transportation Research Board, 80 $0^{\text {th }}$ Annual Meeting, January 7-11, 2001, Washington, D.C., Paper Nº1-0139.

5. Vancaster, N., Moortgat, G., Cambier, F. Caracterización de Productos de Arcilla Prensada Mediante la Espectroscopía de Impedancia Electroquímica.

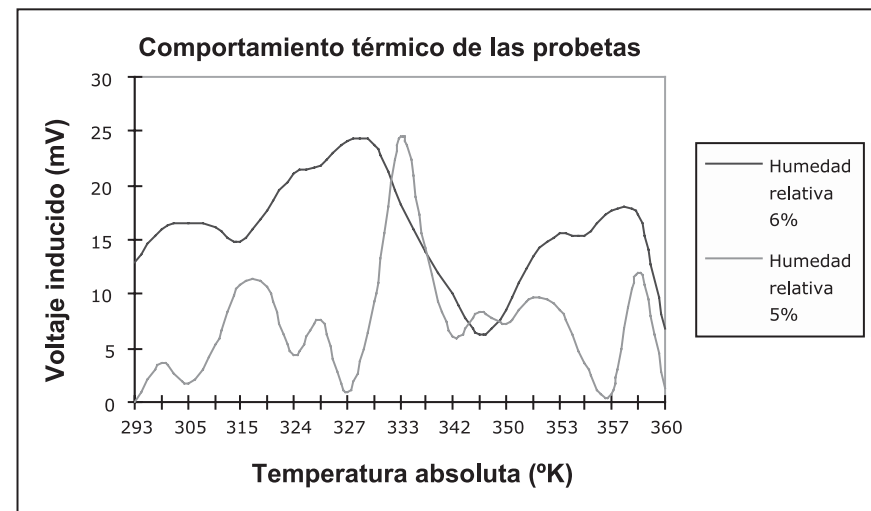

Figura 3. Comportamiento térmico de las probetas de material cerámico crudo

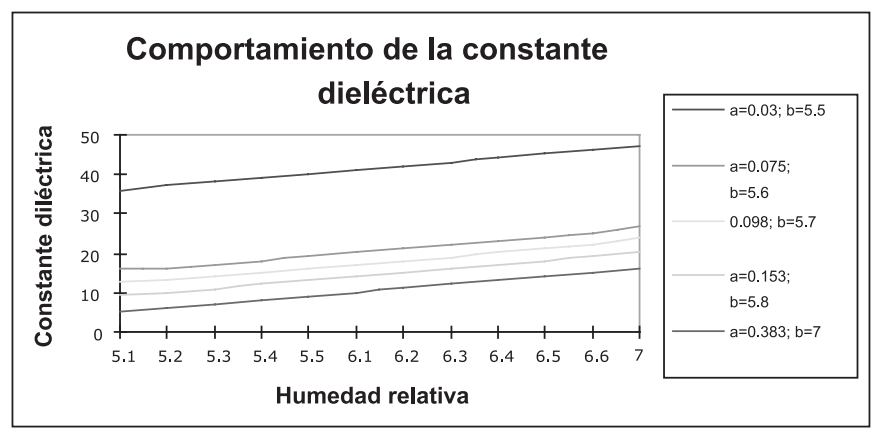

Figura 4. Comportamiento de la constante dieléctrica de las probetas cerámicas con relación a la humedad relativa y factor $\gamma$

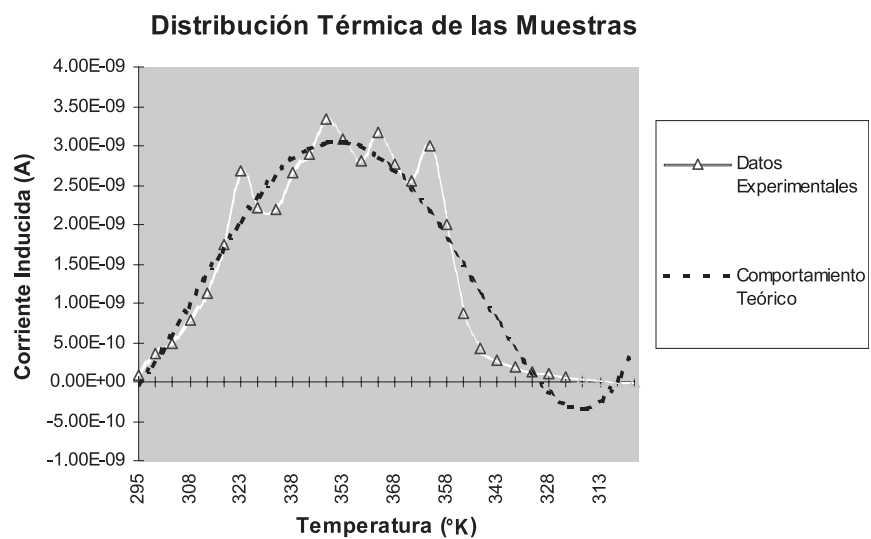

Figura 5. Comportamiento térmico de las muestras con relación a la corriente.

Qualicer 2000. Castellón España. P. GI - 439 - 449.

6. Hammad, S. M., Khalil, A. A., Ghoniem, A., Mohamed, A. I. Some Electrical Properties of the High Dam Lake Sediments. Interceram. Vol. 47. N 6 (1998). Pp. $390-93$

7. D. A., Payne and L. E., Cross, "Microestructure - Property Relations for Dielectric Ceramics: I. Mixing of Isotropic Homogeneous Linear Dielectrics"; pp 584-97 in Ceramic Microestructures 76. Edited by R. M. Fulrath and J. A. Pask. Westview Press, Boulder, CO, 1977.

8. Jackson, J.D., Classical Electrodinamics. Ed. John Wiley \& Sons, Inc. New York - London. 1962

9. Amorós Albaro, J. L., Escardino Benlloch, A., Beltrán Porcar, V., Enrique Navarro, J. E. Control de Compactación durante el Proceso de Fabricación de Pavimentos y Revestimientos Cerámicos. Técnica Cerámica. N ${ }^{\circ} 118$. Pp $1307-14$.

Recibido: 16.10 .02

Aceptado: 30.01 .03 